\title{
Relaxation of multidimensional variational problems with constraints of general form
}

\author{
Farhad Hüsseinov* \\ Department of Economics, Bilkent University, 06533 Bilkent, Ankara, Turkey \\ Received 1 October 1996; accepted 15 July 1999
}

Keywords: Multidimensional variational problem; Relaxation of variational problem; Convex hull; Young-Fenchel conjugate; Piece-wise affine function; Weak convergence

This paper is devoted to further developement of an idea of a well-known theorem of Bogolubov [2]. Here we construct a relaxation of multidimensional variational problems with constraints of rather general form on gradients of admissible functions; it is assumed that the gradient of an admissible function belongs to an arbitrary bounded set. This relaxation involves as a class of admissible functions the closure of the class of admissible functions of the original problem in the topology of uniform convergence, and uses a theorem characterizing this closure, which is proved in [15]. The case when the gradient of an admissible function is constrained within a bounded closed convex body is studied in the works [13,15,19].

The study of multidimensional variational problems was started in 1970s by Ekeland and Temam [13]. The existing literature on relaxation of variational problems, including two monographs by Buttazzo [3] and Dacorogna [9], and the review paper by Marcellini [18] containing a considerable list of references, is quite rich. However, the author failed to find a setting similar to that of the paper. For the most recent results on relaxation and related topics see $[1,4-8,11,14]$.

This paper deals with the case where an integrand depends on a scalar function of several variables. At the end of the paper we will make a conjecture on generalization of the main relaxation result of the paper to the case of an integrand depending on a vector function of several variables. We also make a conjecture on generalization of

* Tel.: +90-04-312-266-4040; fax: +90-04-312-266-4960.

E-mail address: farhad@bilkent.edu.tr (F. Hüsseinov). 
the above-mentioned theorem on characterization of the closure, which is an important tool in the proof of the main result, for the vectorial case.

$R^{n}$ will stand for $n$-dimensional Euclidean space of points $t=\left(t_{1}, \ldots, t_{n}\right)$. Let $\Omega$ be an arbitrary bounded open set in $R^{n}$. Denote by $C(\bar{\Omega})$ the space of all real continuous functions on $\bar{\Omega}$ with the norm

$$
\|x(\cdot)\|_{C(\bar{\Omega})}=\max _{t \in \bar{\Omega}}|x(t)| .
$$

Denote by $W_{\infty}^{1}(\Omega)$ the Sobolev space of all essentially bounded measurable functions on $\Omega$, with essentially bounded first generalized partial derivatives. It is well known that a function $x(\cdot)$ from $W_{\infty}^{1}(\Omega)$ is continuous on $\Omega$ and possesses the ordinary first derivatives $\partial x / \partial t_{i}(i=1, \ldots, n)$ almost everywhere (a.e.) on $\Omega$ (see [13,20]). If domain $\Omega$ satisfies additional conditions (e.g., if $\Omega$ is Lipschitzian), then $W_{\infty}^{1}(\Omega) \subset C(\bar{\Omega})$. Let $\bar{W}_{\infty}^{1}(\Omega)=W_{\infty}^{1}(\Omega) \cap C(\bar{\Omega})$. So, if $\Omega$ is sufficiently regular, then $\bar{W}_{\infty}^{1}(\Omega)=W_{\infty}^{1}(\Omega)$. Denote by $B_{r}(0)$ a ball in $R^{n}$ with the center at the origin and radius $r$. Given a set $V \subset R^{n}$ and a positive number $r$ let $V_{r}=\{v \in V$ : $\operatorname{dist}(v, \partial V) \geq r\}$, where $\partial V$ is the boundary of $V$.

Recall that function $x(\cdot): \bar{\Omega} \rightarrow R$ is said to be piece-wise affine, if it is continuous and there exists a partition of $\bar{\Omega}$ into a subset of measure zero and a finite number of open sets, on which $x(\cdot)$ is affine. A continuous function on $\bar{\Omega}$ is said to be almost piece-wise affine, if its restriction to an arbitrary strict interior subdomain of $\Omega$ is piece-wise affine.

Let $X, Y$ be topological spaces, and $I, J$ be functionals defined on $X$ and $Y$, respectively. The variational problem $\inf \{J(y): y \in Y\}$ is said to be a relaxation of the problem $\inf \{I(x): x \in X\}$, if there exists a continuous mapping $i: X \rightarrow Y$, such that: (i) $i(X)$ is dense in $Y$, (ii) $J(i(x)) \leq I(x)$ for each $x \in X$, and (iii) for an arbitrary $y \in Y$ there exists a sequence $x_{k} \in X(k \in N)$ such that $i\left(x_{k}\right) \rightarrow y$ and $J(y) \geq \lim _{k \rightarrow \infty} I\left(x_{k}\right)$. Moreover, if functional $J$ is lower semicontinuous, then a relaxation is called a lower semicontinuous relaxation (see [16]).

Let $f: \bar{\Omega} \times R \times R^{n} \rightarrow R$ be a continuous function, $U$ be an arbitrary bounded set in $R^{n}$ with an affine hull $R^{n}, \Gamma \subset \partial \Omega$ and $\phi: \Gamma \rightarrow R$ be some fixed function. Consider the following problem of multidimensional variational calculus, which we will refer to as problem $(\mathrm{P})$ :

$$
\begin{aligned}
& J(x(\cdot))=\int_{\Omega} f(t, x(t), \operatorname{grad} x(t)) d(t) \rightarrow \inf , \\
& \operatorname{grad} x(t) \in U \quad \text { a.e. in } \Omega, \\
& x(t)=\phi(t) \quad \text { for } t \in \Gamma,
\end{aligned}
$$

where $x(\cdot) \in \bar{W}_{\infty}^{1}(\Omega)$. The case when $\Gamma=\emptyset$, i.e., when the boundary condition (3) is absent, will be referred to as problem $\left(\mathrm{P}_{0}\right)$.

A function $x(\cdot) \in \bar{W}_{\infty}^{1}(\Omega)$ is called admissible in problem $(\mathrm{P})\left(\left(\mathrm{P}_{0}\right)\right)$, if it satisfies conditions $(2),(3)((2))$. The set of all admissible functions in problem $(\mathrm{P})\left(\left(\mathrm{P}_{0}\right)\right)$ will 
be denoted by $E(U, \phi)(E(U))$. Thus

$$
\begin{aligned}
& E(U)=\left\{x(\cdot) \in \bar{W}_{\infty}^{1}(\Omega): \operatorname{grad} x(t) \in U \text { a.e. in } \Omega\right\}, \\
& E(U, \phi)=\left\{x(\cdot) \in E(U):\left.x(\cdot)\right|_{\Gamma}=\phi\right\} .
\end{aligned}
$$

The space $\bar{W}_{\infty}^{1}(\Omega)$ and its subsets $E(U), E(U, \phi)$ will be considered with the metric of uniform convergence.

Along with problem $(\mathrm{P})$ we consider the following problem (problem $(\mathrm{PR})$ ):

$$
\begin{aligned}
& J_{R}(x(\cdot))=\int_{\Omega} f_{U}^{* *}(t, x(t), \operatorname{grad} x(t)) d(t) \rightarrow \inf , \\
& x(t)=\phi(t) \quad \text { for } t \in \Gamma,
\end{aligned}
$$

where $\overline{c o} U$ is the closed convex hull of $U$ and $f_{U}^{* *}(t, x, \cdot)=(f(t, x, \cdot)+\delta(\cdot \mid U))^{* *}$. Here

$$
\delta(u \mid U)= \begin{cases}0 & \text { for } u \in U, \\ +\infty & \text { for } u \in R^{n} \backslash U\end{cases}
$$

is the indicator function of $U$, and ${ }^{* *}$ designates the operation of taking second YoungFenchel conjugate (see [17, p. 183]). In case of $\Gamma=\emptyset$ problem (PR) will be denoted as $\left(\mathrm{P}_{0} \mathrm{R}\right)$.

The above-mentioned assertion on closure consists of the following:

$$
\overline{E(U)}=E(\overline{c o} U)
$$

i.e. the closure in the uniform metric of a class of functions continuous on $\Omega$ with gradients from the bounded set $U$ coincides with the class of functions continuous on $\bar{\Omega}$ and with gradients from the closed convex hull of $U$. Moreover, if condition (4) of Theorem 1 below is satisfied, then Theorem $1^{\prime}$ from Hüsseinov [15] implies the following coincidence

$$
\overline{E(U, \phi)}=E(\overline{c o} U, \phi) \text {. }
$$

Theorem 1. Let $U \subset R^{n}$ be an arbitrary bounded set in $R^{n}$ with the affine hull $R^{n}$. Suppose that there exists an admissible function $y_{0}(\cdot) \in E(\overline{c o} U, \phi)$ in problem $(\mathrm{PR})$ such that

$$
\operatorname{grad} y_{0}(t) \in U_{0} \text { a.e. in } \Omega \text {, }
$$

where $U_{0}$ is a closed set contained in the interior of $\overline{c o} U$. Then, for an arbitrary function $x(\cdot) \in E(\overline{c o} U, \phi)$ admissible in problem (PR), there exists a sequence of functions $x_{k}(\cdot)(k \in N)$, admissible in problem $(\mathrm{P})$, uniformly converging to $x(\cdot)$, and such that

$$
\lim _{k \rightarrow \infty} J\left(x_{k}(\cdot)\right)=J_{R}(x(\cdot)) .
$$

In particular, when the boundary condition (3) is absent, i.e. for problem $\left(\mathrm{P}_{0}\right)$, condition (4) in Theorem 1 is satisfied automatically. 
The following lemma will be used in the proof of Theorem 1 .

Lemma. Let $T$ be a topological space, $U$ be an arbitrary bounded set in $R^{n}, U_{0} \subset U$ be a compact set contained in the interior of $\overline{c o} U$ or a segment, and $f: T \times R^{n} \rightarrow R$ be a continuous function. Then a restriction of function $f_{U}^{* *}(\tau, u)$ to $T \times U_{0}$ is continuous.

Proof. Since $f_{U}^{* *}=f_{\bar{U}}^{* *}$, we suppose, without loss of generality, that $U$ is closed. Fix a point $\left(\tau_{0}, u_{0}\right) \in T \times U_{0}$ and a positive number $\varepsilon$. It is easily seen that, there exists a neighborhood $S\left(\tau_{0}\right)$ of point $\tau_{0}$ such that

$$
\left|f(\tau, u)-f\left(\tau_{0}, u\right)\right|<\varepsilon \quad \text { for } \tau \in S\left(\tau_{0}\right), \quad u \in \overline{c o} \bar{U} .
$$

It is well known that

$$
f_{U}^{* *}=\min \left\{\sum_{i=1}^{n+1} \lambda_{i} f\left(\tau, u_{i}\right): \sum_{i=1}^{n+1} \lambda_{i} u_{i}=u, u_{i} \in U, \sum_{i=1}^{n+1} \lambda_{i}=1, \lambda_{i} \geq 0\right\} .
$$

From this and (5) we obtain that

$$
f_{U}^{* *}(\tau, u)=\sum_{i=1}^{n+1} \bar{\lambda}_{i} f\left(\tau, \bar{u}_{i}\right) \geq \sum_{i=1}^{n+1} \bar{\lambda}_{i} f\left(\tau_{0}, \bar{u}_{i}\right)-\varepsilon \geq f_{U}^{* *}\left(\tau_{0}, u\right)-\varepsilon .
$$

Symmetrically,

$$
f_{U}^{* *}\left(\tau_{0}, u\right) \geq f_{U}^{* *}(\tau, u)-\varepsilon .
$$

Consequently,

$$
\left|f_{U}^{* *}\left(\tau_{0}, u\right)-f_{U}^{* *}\left(\tau_{0}, u\right)\right|<\varepsilon \quad \text { for } \tau \in S\left(\tau_{0}\right) \quad u \in \overline{c o} U .
$$

Since $f_{U}^{* *}\left(\tau_{0}, \cdot\right)$ is a convex and lower semicontinuous it is continuous on $U$ (in both the cases stipulated in the lemma). Therefore, there exists a number $\delta>0$ such that

$$
\left|f_{U}^{* *}(\tau, u)-f_{U}^{* *}\left(\tau_{0}, u_{0}\right)\right|<\varepsilon \quad \text { for } u \in U_{0}, \quad\left\|u-u_{0}\right\|<\delta .
$$

The last two inequalities imply that

$$
\left|f_{U}^{* *}(\tau, u)-f_{U}^{* *}\left(\tau_{0}, u_{0}\right)\right|<2 \varepsilon
$$

for $\tau \in S\left(\tau_{0}\right),\left\|u-u_{0}\right\|<\delta$. Therefore, function $\left.f_{U}^{* *}\right|_{T \times U_{0}}$ is continuous at the point $\left(\tau_{0}, u_{0}\right)$.

Proof of Theorem 1. Let $x(\cdot) \in E(\overline{c o} \bar{U}, \phi)$ be an admissible function in problem $(\mathrm{PR})$ and $\varepsilon>0$. Consider the sequence of functions $x_{k}(t)=((k-1) / k) x(t)+(1 / k) y_{0}(t)$ $(k \in N)$. Clearly, $x_{k}(\cdot) \in E(\overline{c o} U, \phi)$ and

$$
\begin{aligned}
& x_{k}(\cdot) \rightarrow_{k} x(\cdot) \quad \text { uniformly on } \Omega, \\
& \operatorname{grad} x_{k}(t) \rightarrow_{k} \operatorname{grad} x(t) \quad \text { for a.a. } t \in \Omega, \\
& \operatorname{grad} x_{k}(t)+B_{r_{k}}(0) \subset U \quad \text { for a.a. } t \in \Omega,
\end{aligned}
$$

where $r_{k},(k \in N)$ are positive numbers. 
It follows from relations (6), (8) and the lemma that

$$
\begin{aligned}
& \left\|x_{k}(\cdot)-x(\cdot)\right\|_{C(\bar{\Omega})}<\frac{\varepsilon}{4}, \\
& \left|J_{R}\left(x_{k}(\cdot)\right)-J_{R}(x(\cdot))\right|<\frac{\varepsilon}{4}
\end{aligned}
$$

for sufficiently large indices $k$.

Let $k_{0}$ be such that (9) holds for $k_{0}$. Let $\bar{x}(\cdot)=x_{k_{0}}(\cdot), r=r_{k_{0}} / 2$. By Theorem $1^{\prime}$ from Hüsseinov [15], there exists a sequence of almost piece-wise affine functions $y_{k}(\cdot) \in E(\overline{c o} U, \phi)$ uniformly converging to $x(\cdot)$. Then the sequence of vector functions $\operatorname{grad} y_{k}(\cdot)(k \in N)$ weakly converges to vector function $\operatorname{grad} \bar{x}(\cdot)$ in Banach space $L_{1}^{n}(\Omega)$ of summable $n$-vector functions on domain $\Omega$. By Mazur's Theorem (Corollary 3.14 from Dunford and Schwartz [12, p. 457]) it follows that there exist convex combinations $z_{m}(\cdot)=\sum_{k=N_{m}+1}^{N_{m+1}} \alpha_{k}^{m} y_{k}(\cdot)(m \in N)$ of functions $y_{k}(\cdot)(k \in N)$, where $\alpha_{k} \geq 0, \sum_{k=N_{m}+1}^{N_{m+1}} \alpha_{k}^{(m)}=1$ and $N_{m}(m \in N)$ is a strictly increasing sequence of integers such that

$$
\operatorname{grad} z_{m}(t) \rightarrow \operatorname{grad} \bar{x}(t) \quad \text { for a.a. } t \in \Omega .
$$

Thus, the functions $z_{m}(\cdot)$ are almost piece-wise affine, $z_{m}(\cdot) \in E\left((\overline{c o} U)_{r}, \phi\right)(m=$ $1,2, \ldots)$, the sequence $z_{m}(\cdot)(m \in N)$ uniformly converges to $\bar{x}(\cdot)$, and condition $(10)$ is satisfied. From that we obtain

$$
\begin{aligned}
& \mid\left\|z_{m}(\cdot)-\bar{x}(\cdot)\right\|_{C(\bar{\Omega})}<\frac{\varepsilon}{4}, \\
& \left|J_{R}\left(z_{m}(\cdot)\right)-J_{R}(\bar{x}(\cdot))\right|<\frac{\varepsilon}{4}
\end{aligned}
$$

for sufficiently large $m$. Fix one of such indices $m_{0}$ and denote $\bar{z}(\cdot)=z_{m_{0}}(\cdot)$. We obtain from relations (9) with $k=k_{0}$ and (11) with $m=m_{0}$

$$
\begin{aligned}
& \|\bar{z}(\cdot)-x(\cdot)\|_{C(\Omega)}<\frac{\varepsilon}{2}, \\
& \left|J_{R}(\bar{z}(\cdot))-J_{R}(x(\cdot))\right|<\frac{\varepsilon}{2} .
\end{aligned}
$$

So, function $\bar{z}(\cdot)$ is almost piece-wise affine, $\bar{z}(\cdot) \in E\left((\overline{c o} U)_{r}, \phi\right)$ and satisfies relations (12).

Denote $M=1+\max |x(t)|$. Since integrand $f$ is continuous on compact $K=\bar{\Omega} \times$ $[-M, M] \times \bar{U}$, there exists a positive number $\delta_{0}^{\prime}<\varepsilon / 2$ such that

$$
\left|f\left(t_{1}, x_{1}, u_{1}\right)-f\left(t_{2}, x_{2}, u_{2}\right)\right|<\frac{\varepsilon}{2}
$$

for $\left(t_{1}, x_{1}, u_{1}\right),\left(t_{2}, x_{2}, u_{2}\right) \in K,\left\|t_{1}-t_{2}\right\|<\delta_{0}^{\prime},\left\|u_{1}-u_{2}\right\|<\delta_{0}^{\prime}$.

In sequel, we shall omit the index $U$ in notation $f_{U}^{* *}$. By the lemma function $f^{* *}$ is continuous on compact $K_{r}=\bar{\Omega} \times[-M, M] \times(\overline{c o} U)_{r}$. Hence, there exists $\delta_{0} \in\left(0, \delta_{0}^{\prime}\right)$ such that

$$
\left|f^{* *}\left(t_{1}, x_{1}, u_{1}\right)-f^{* *}\left(t_{1}, x_{1}, u_{1}\right)\right|<\frac{\varepsilon}{2}
$$


for $\left(t_{1}, x_{1}, u_{1}\right),\left(t_{2}, x_{2}, u_{2}\right) \in K_{r}{ }^{\prime}\left\|t_{1}-t_{2}\right\|<\delta_{0},\left\|u_{1}-u_{2}\right\|<\delta_{0}$. Since the functions $x(\cdot)$ and $\bar{z}(\cdot)$ are continuous on $\Omega$, there exists $\delta \in\left(0, \delta_{0} / 2\right)$ such that

$$
\left|x\left(t_{1}\right)-x\left(t_{2}\right)\right|<\delta_{0}, \quad\left|\bar{z}\left(t_{1}\right)-\bar{z}\left(t_{2}\right)\right|<\frac{\delta_{0}}{2} \quad \text { for }\left\|t_{1}-t_{2}\right\|<\delta .
$$

Denote by $\Delta_{j}(j \in N)$ the simplices of affineness of function $\bar{z}(\cdot), a_{j}=\operatorname{grad} z(t)$ for $t \in$ int $\Delta_{j}(j \in N)$. Without loss of generality, we assume that diam $\Delta_{j}<\delta(j \in N)$. Fix $t_{j} \in \Delta_{j}(j \in N)$. It is well known that

$$
\begin{aligned}
f^{* *} & \left(t_{j}, \bar{z}\left(t_{j}\right), a_{j}\right) \\
& =\inf \left\{\sum_{i=1}^{n+1} \alpha_{i}^{j} f\left(t_{j}, \bar{z}\left(t_{j}\right), v_{i}^{j}\right): \sum_{i=1}^{n+1} \alpha_{i}^{j} v_{i}^{j}=a_{j}, v_{i}^{j} \in U, \sum_{i=1}^{n+1} \alpha_{i}^{j}=1, \alpha_{i}^{j} \geq 0\right\} .
\end{aligned}
$$

Then for some numbers $\alpha_{i}^{j}>0(i=1,2, \ldots, n+1), \sum_{i=1}^{n+1} \alpha_{i}^{j}=1$ and affinely independent vectors $v_{i}^{j}(i=1,2, \ldots, n+1)$ from $U$

$$
\begin{aligned}
& \left|f^{* *}\left(t_{j}, \bar{z}\left(t_{j}\right), a_{j}\right)-\sum_{i=1}^{n+1} \alpha_{i}^{j} f\left(t_{j}, \bar{z}\left(t_{j}\right), v_{i}^{j}\right)\right|<\frac{\varepsilon}{2}, \\
& \sum_{i=1}^{n+1} \alpha_{i}^{j} v_{i}^{j}=a_{j} .
\end{aligned}
$$

Put $u_{i}^{j}=v_{i}^{j}-a_{j}(i=1,2, \ldots, n+1)$ and denote $\sum_{j}=\operatorname{co}\left\{u_{1}^{j}, \ldots, u_{n+1}^{j}\right\}$. Since, vectors $u_{i}^{j}(i=1,2, \ldots, n+1)$ are affinely independent and $\sum_{i=1}^{n+1} \alpha_{i}^{j} v_{i}^{j}=0$, where $\alpha_{i}^{j}>0(i=$ $1,2, \ldots, n+1)$ then $\sum_{j}$ is an $n$-dimensional simplex with the interior containing zero. Denote $D_{j}=\sum_{j}^{0}$ polar of the simplex $\sum_{j}, s_{j}(\cdot)-$ support function of set $\left\{u_{1}^{j}, \ldots, u_{n+1}^{j}\right\}$.

Partition simplex $\Delta_{j}$ into at most countably many simplices $\Delta_{1}^{j}, \Delta_{2}^{j}, \ldots$, homothetic to $D_{j}$ and such that $\operatorname{diam} \Delta_{k}^{j}<\delta \operatorname{diam} D_{j}$. Denote by $d_{k}^{j}$ the similarity coefficients of simplices $\Delta_{k}^{j}$ and $D_{j}$ and put

$$
s_{k}^{j}(t)= \begin{cases}s\left(t-t_{k}^{j}\right)-d_{k}^{j} & \text { for } t \in \Delta_{k}^{j}, \\ 0 & \text { for } t \in \bar{\Omega} \backslash \Delta_{k}^{j}\end{cases}
$$

and $\sigma_{i}\left(\Delta_{k}^{j}\right)=\left\{t \in \Delta_{k}^{j}: s_{k}^{j}(t)=\left\langle t-t_{k}^{j}, u_{k}^{j}\right\rangle-d_{k}^{j}\right\}(i=1,2, \ldots, n+1)$, for arbitrary indices $j, k$, where $t_{k}^{J} \in \Delta_{k}^{j}$ is the image of the origin under the homothety $D_{j} \rightarrow \Delta_{k}^{j}$. Obviously, function $s_{k}^{j}(\cdot)$ is piece-wise affine and

$$
-\delta \leq s_{k}^{j}(t) \leq 0 \text {. }
$$

Put

$$
s(t)=\sum_{j, k} s_{k}^{j}(t) \quad \text { and } \quad z(t)=\bar{z}(t)+s(t) .
$$

Since

$$
\operatorname{grad} z(t)=\operatorname{grad} \bar{z}(t)+u_{i}^{j}=a_{j}+u_{i}^{j}=v_{i}^{j} \in U \quad \text { for } t \in \sigma_{i}\left(\Delta_{k}^{j}\right)
$$


and simplices $\sigma_{i}\left(\Delta_{k}^{j}\right)(i=1,2, \ldots, n+1 ; j, k \in N)$ cover domain $\Omega$, then function $z(\cdot)$ is admissible in problem $(\mathrm{P})$, i.e. $z(\cdot) \in E(U, \phi)$.

Utilizing inequalities (15)-(17) and Proposition 2 from Hüsseinov [15] we estimate the difference

$$
\begin{aligned}
& \left.\mid \int_{\Delta_{k}^{j}} f^{* *}(t, \bar{z}(t), \operatorname{grad} \bar{z}(t)) \mathrm{d}(t)-\int_{\Delta_{k}^{j}} f(t, z(t), \operatorname{grad} z(t)) \mathrm{d} t\right) \mid \\
& \quad=\left|\int_{\Delta_{k}^{j}} f^{* *}(t, \bar{z}(t), \operatorname{grad} \bar{z}(t)) \mathrm{d}(t)-\sum_{i+1}^{n+1} \int_{\sigma_{i}\left(\Delta_{k}^{j}\right)} f\left(t, \bar{z}(t)+s_{k}^{j}(t), v_{i}^{j}\right) \mathrm{d}(t)\right| \\
& \quad \leq\left|\operatorname{mes}\left(\Delta_{k}^{j}\right) f^{* *}\left(t_{j}, \bar{z}\left(t_{j}\right), a_{j}\right)-\sum_{i=1}^{n+1} \alpha_{i}^{j} \operatorname{mes}\left(\Delta_{k}^{j}\right) f\left(t_{j}, \bar{z}\left(t_{j}\right), v_{i}^{j}\right)\right|+\varepsilon \operatorname{mes}\left(\Delta_{k}^{j}\right) \mid \\
& \quad=\operatorname{mes}\left(\Delta_{k}^{j}\right)\left[\left|f^{* *}\left(t_{j}, \bar{z}\left(t_{j}\right), a_{j}\right)-\sum_{i=1}^{n+1} \alpha_{i}^{j} f\left(t_{j}, \bar{z}\left(t_{j}\right), v_{i}^{j}\right)\right|\right] \leq 2 \varepsilon \operatorname{mes}\left(\Delta_{k}^{j}\right) .
\end{aligned}
$$

Summing up inequalities (18) by $j, k$ we obtain

$$
\left|J_{f^{* *}}(\bar{z}(\cdot))-J(\bar{z}(\cdot))\right|<2 \varepsilon \operatorname{mes}(\Omega) .
$$

It is clear from (17) that

$$
\|\bar{z}(\cdot)-z(\cdot)\|_{C(\bar{\Omega})}<\frac{\varepsilon}{2} .
$$

From this and from the first of inequalities (12) it follows that

$$
\|z(\cdot)-x(\cdot)\|_{C(\bar{\Omega})}<\varepsilon,
$$

and from (19) and from the second of inequalities (12) that

$$
\left|J_{R}(x(\cdot))-J(z(\cdot))\right|<\varepsilon[1+2 \operatorname{mes}(\Omega)] .
$$

The theorem is proved.

Theorem 1 and Lemma 4 from Hüsseinov [15] imply the following result.

Theorem 2. Let $U$ be a bounded set in $R^{n}$ with an affine hull $R^{n}$, and assumption (4) of Theorem 1 be satisfied. Then problem (PR) is a lower semicontinuous relaxation of problem $(\mathrm{P})$.

For $U \subset R^{m \times n}$ the closure of the quasiconvex hull is defined as (see [10, Definition 2.2]):

$$
\overline{Q c o} U=\left\{\xi \in R^{m \times n}: f(\xi) \leq 0, \forall f: R^{m \times n} \rightarrow R, \text { quasiconvex and }\left.f\right|_{U}=0\right\} .
$$

We denote for $U \subset R^{m \times n}$

$$
E(U)=\left\{x(\cdot) \in W_{\infty}^{1}\left(\Omega ; R^{m}\right): D x(t) \in U \text { a.e. in } \Omega\right\},
$$


where $D x(t)$ denotes the Jacobi matrix of $x(\cdot)$ at $t$. We conjecture the following coincidence: $\overline{E(U)}=E(\overline{Q c o} U)$, where $\overline{E(U)}$ denotes the closure of $E(U)$ in uniform metric of $\bar{W}_{\infty}^{1}\left(\Omega ; R^{m}\right)$.

Consider the following two variational problems. The first is the problem $(\mathscr{P})$ obtained from (P) by treating $f$ as a function $R^{m \times n} \rightarrow R$, $\operatorname{grad} x(t)$ replaced by $D x(t)$ the Jacobi matrix of $x(\cdot): \Omega \rightarrow R^{m}$ at $t$, and $\phi(\cdot): \Gamma \rightarrow R^{m}$. The second problem is

$$
\begin{aligned}
& J_{R}(x(\cdot))=\int_{\Omega} Q f_{U}(t, x(t), D x(t)) \mathrm{d} t \rightarrow \mathrm{inf}, \\
& x(t)=\phi(t) \quad \text { for } t \in \Gamma,
\end{aligned}
$$

where $Q f_{U}(t, x, \cdot)$ is the quasiconvex envelope (i.e. the maximal quasiconvex function not exceeding $f$ ) of the function $f(t, x, \cdot)+\delta(\cdot \mid U), \delta(\cdot \mid U)$ being the indicator function of $U$.

Conjecture. Let $U \subset R^{m \times n}$ be an arbitrary bounded set with $\overline{Q c o} U$ having an interior point. Suppose that there exists an admissible function $y_{0}(\cdot) \in E(\overline{Q c o} U, \varphi)$ in problem $(\mathscr{P} R)$ such that $D y_{0}(t) \in U_{0}$ a.e. in $\Omega$, where $U_{0}$ is a closed set contained in the interior of $\overline{Q c o} U$, then for an arbitrary vector function $x(\cdot) \in E(\overline{Q c o} U, \varphi)$ admissible in problem $(\mathscr{P} R)$, there exists a sequence of vector-functions $x_{k}(\cdot)(k \in N)$ admissible in problem $(\mathscr{P})$, uniformly converging to $x(\cdot)$ and such that

$$
\lim J\left(x_{k}(\cdot)\right)=J_{R}(x(\cdot)) .
$$

\section{Acknowledgements}

I am grateful to an anonymous referee for helpful comments.

\section{References}

[1] L. Ambrosio, G. Dal Maso, On the relaxation in $B V\left(\Omega ; R^{m}\right)$ of quasi-convex integrals, J. Funct. Anal. 109 (1992) 1.

[2] N.N. Bogolubov, Sur quelques methods dans la calculus des variations, Amer. Math. Pure. Ser. 4 (1930) 249-271.

[3] G. Buttazzo, Semicontinuity, Relaxation, and Integral Representations in the Calculus of Variations, Longman Scientific and Technical, Harlow, 1989.

[4] G. Bouchitte, G. Buttazzo, Relaxation for a class of nonconvex functionals defined on measures, Ann. Inst. H. Poincare, Anal. Non Linearie 10 (1993) 345-361.

[5] G. Buttazzo, G. Dal Maso, An existence result for a class of shape optimization problems, Arch. Rational Mech. Anal. 122 (1993) 183-195.

[6] G. Buttazzo, G. Dal Maso, A. Garonni, A. Malusa, On relaxed formulation of shape optimization problems, Adv. Math. Sci. Appl. 7 (1997) 1-24.

[7] L. Carbone, R. De Arcangelis, On the relaxation of some classes of unbounded integral functionals, Matematiche (Catania) 51 (1996) 221-252.

[8] E.A. Corbo, R. De Arcangelis, Comparison results for some types of relaxation of variational integral functionals, Ann. Mat. Pura Appl. 164 (1993) 155-193.

[9] B. Dacorogna, Direct Methods in the Calculus of Variations, Springer, New York, 1989. 
[10] B. Dacorogna, P. Marcellini, Cauchy-Dirichlet problem for first order nonlinear systems, J. Funct. Anal. 152 (1998) 404-446.

[11] R. De Arcangelis, L. Carbone, On integral representation, relaxation and homogenization for unbounded functionals, Atti Accad. Naz. Lincei, Mat. Appl. 8 (1997) 129-135.

[12] N. Dunford, J.T. Schwartz, Linear Operators, Vol. 1, Interscience Publishers, New York, 1958.

[13] I. Ekeland, R. Temam, Convex Analysis and Variational Problems, North-Holland, Amsterdam, 1976.

[14] I. Fonseca, P. Marcellini, Relaxation of multiple integrals in subcritical Sobolev Spaces, J. Geom. Anal. 7 (1997) 57-81.

[15] F.V. Hüsseinov, On the question of extension of multidimensional variational problems, Izv. Acad. Sci. USSR, Ser. Math. 50 (1986) 3-21; English transl., Math. USSR Izv. 28 (1987) 1-19.

[16] A.D. Ioffe, V.M. Tikhomirov, Relaxation of Variational Problems, Trudy Moskov. Mat. Obshch. 18 (1968) 187-246.

[17] A.D. Ioffe, V.M. Tikhomirov, Theory of Extremal Problems, Nauka, Moscow, 1974.

[18] P. Marcellini, Non convex integrals of the calculus of variations, in: A. Cellina (Ed.), Methods of Nonconvex Analysis (Varenna 1989), Lecture Notes in Mathematics, Vol. 1446, Springer, Berlin, 1990 16-57.

[19] V.I. Matov, Study of one problem of multidimensional variational problem, Viestnik MGU, Ser. Mat. Mech. 1 (1978) 61-69.

[20] S.L. Sobolev, Some Applications of Functional Analysis in Mathematical Physics, LGU, Leningrad, 1959. 